\title{
The collisionality dependence of intermittency level in drift-wave turbulence in the stellarator TJ-K
}

\author{
S. Garland, ${ }^{1}$ K. Reuther, ${ }^{1}$ M. Ramisch, ${ }^{1}$ and P. Manz ${ }^{2}$ \\ 1) Institute of Interfacial Process Engineering and Plasma Technology, \\ University of Stuttgart, 70569 Stuttgart, Germany \\ ${ }^{2)}$ Max Planck Institute for Plasma Physics, 85748 Garching, \\ Germany
}

(Dated: 1 February 2018)

The link between plasma collisionality and the intermittency of edge drift-wave turbulence is investigated experimentally in the stellarator TJ-K and compared to results obtained from slab Hasegawa-Wakatani (HW) simulations. The kurtosis of experimental time series of density and potential fluctuations was analysed at different frequency scales. Using this method on data from a range of discharges, it was found that the intermittency level increases with increasing collisionality for density fluctuations, whereas potential fluctuations are generally self-similar. In addition, a high-order structure function analysis of density time-series data also shows a trend towards higher intermittency levels as collisionality is increased. HW simulations are found to produce a qualitatively similar trend in the intermittency level compared to experimental analyses. The transition to intermittent fluctuations at higher collisionalities can be understood in the framework of the HW model as the decoupling of the density field from the self-similar potential field when moving from the adiabatic to the hydrodynamic limit, where the density behaves like a passive scalar, resulting in the intermittency levels observed in the vorticity. 


\section{INTRODUCTION}

In magnetically confined fusion plasmas, radial transport of particles and heat limits the achievable confinement times and poses a threat to the reactor's main chamber walls through high associated heat loads ${ }^{1}$. A significant proportion of the transport in the scrape-off layer (SOL) to the main chamber walls can be due to intermittent filamentary structures, often referred to as blobs ${ }^{2}$. The filamentary transport in the scrape-off layer depends strongly on the collisionality ${ }^{3}$ and the effect of collisionality on the sizes and velocities of the filaments can be understood ${ }^{4}$. The collisonality dependence of the intermittency of SOL density fluctuations is, however, not yet clear. Blob filaments are thought to be generated in the vicinity of the separatrix ${ }^{2,5}$, and so it is in this region that the origin of intermittency should be investigated. For low beta edge plasmas, near the separatrix the turbulence is largely due to electrostatic drift-waves ${ }^{6}$. Electrostatic drift-wave turbulence is quasi two-dimensional and in two-dimensional turbulence the velocity field is non-intermittent ${ }^{7}$. The collisionality dependence of the intermittency level of density fluctuations in this region is, on the other hand, an open question.

Due to its relative simplicity, the 2D Hasegawa-Wakatani (HW) model ${ }^{8,9}$ is often chosen to study drift-wave turbulence in the edge region (for example Refs. 10-14). The density fluctuations at the edge of magnetically confined fusion plasmas have been found to have intermittent behaviour ${ }^{13-15}$, whereas potential fluctuations are generally found to be selfsimilar $^{13,14}$. Lagrangian velocity increments in HW simulations have been found to display increasingly intermittent behaviour with increasing collisionality ${ }^{12,16}$. Kadoch et al ${ }^{12}$ show that for small values of collisionality, exponential tails are observed in the Lagrangian acceleration, and the flow is determined by a Gaussian-like diffusion process, however for large values of collisionality, the PDFs obtain heavy tails reflecting the presence of intermittency and indicating a faster than diffusive transport.

The stellarator $\mathrm{TJ}-\mathrm{K}^{17}$ provides a well suited environment to test experimentally for scalings of turbulence with control parameters such as collisionality. A range of working gases is available, allowing the collisionality to be varied over a relatively wide range ${ }^{18,19}$. In addition, the low densities and temperatures allow Langmuir probes to be used inside the confined region, where the turbulence has a drift-wave character ${ }^{20-22}$, and therefore relatively long time series measurements may be taken to access higher-order statistics. 
The aim of this work is to contribute evidence for the collisionality dependence of the intermittency level of the key plasma quantities of electron density and plasma potential from experiments at the stellarator TJ-K, as well as to quantitatively compare the experimental analyses to predictions from HW simulations. The paper is laid out as follows. First of all, two different methods for quantifying the intermittency level are presented. Following this the HW model is briefly explained, along with the parameters used to simulate data in the range required for comparison with experiment. The experimental setup is then introduced along with the Langmuir probe diagnostic, used to obtain experimental time series. The results are divided into two sections, corresponding to the two different analysis techniques for determining the intermittency level. In the first part, a scale dependent kurtosis analysis is used to provide an initial insight into the intermittency of experimental time series. In the second section, a structure function method for the quantification of intermittency is used and the experimental results are compared to the HW simulation data.

\section{CHARACTERISING INTERMITTENCY}

Intermittency can be thought of roughly as the scale dependence of the statistical properties of a signal. It may be quantified mathematically in various ways, two of which are employed here for experimental data. Whilst in the field of neutral fluids, spatial intermittency is frequently studied, in plasma physics, experimental limitations often confine study of turbulence to the time domain. However, a comparison with spatial intermittency can be made if the Taylor approximation ${ }^{23}$ is invoked. U. Frisch proposed ${ }^{24}$ that a time series $x(t)$ is intermittent if the kurtosis of the high-pass-filtered signal $x_{f}^{>}(t)$ increases without bound with cut-off frequency, $f$. The kurtosis is calculated as the flatness minus three,

$$
K_{f}=\frac{\left\langle\left(x_{f}^{>}(t)\right)^{4}\right\rangle}{\left\langle\left(x_{f}^{>}(t)\right)^{2}\right\rangle^{2}}-3,
$$

where $\langle\ldots\rangle$ represents a statistical average. The probability density functions (PDFs) of the fluctuating part of a high-pass filtered electron density signal, obtained from Langmuir probe measurements, along with $10 \mathrm{~ms}$ sections of the time series, are shown in Fig. 1 for three 
a) $\mathrm{n}[\sigma]$
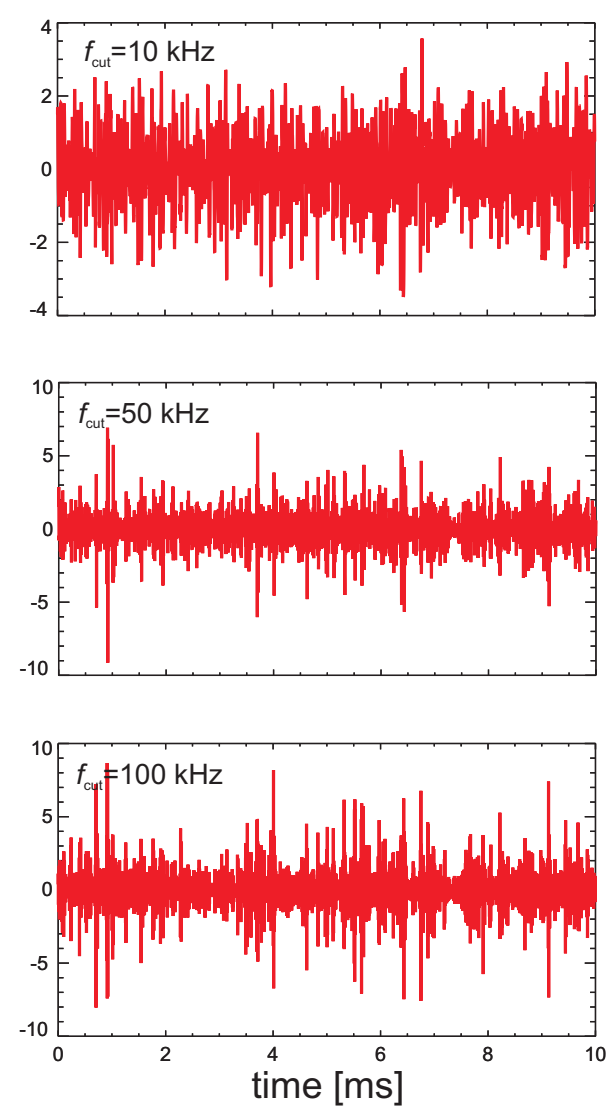

b) PDF

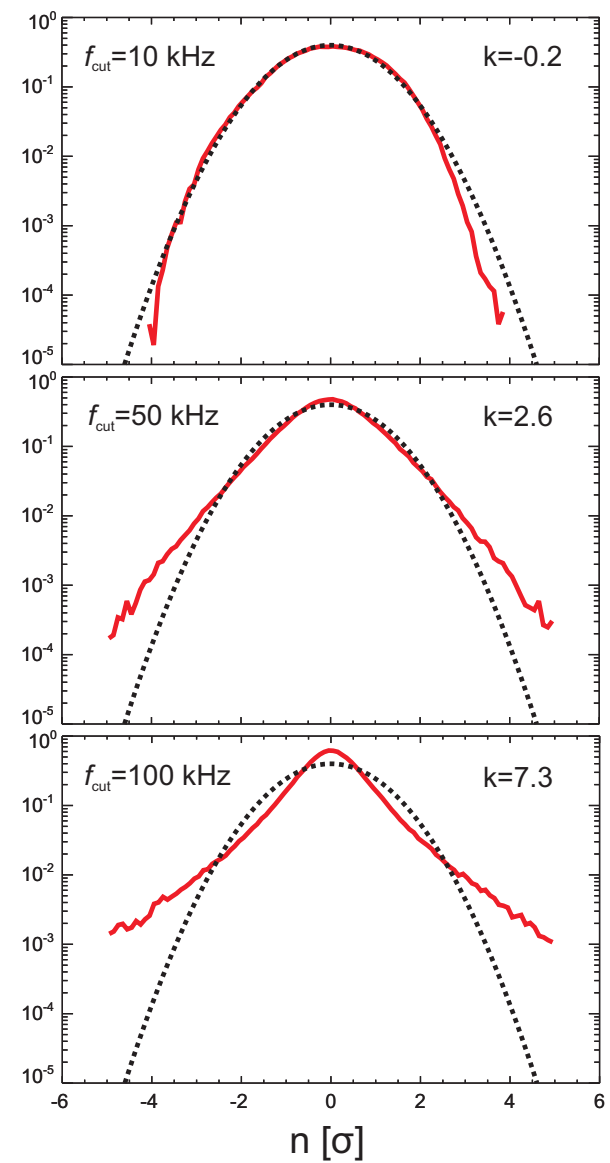

FIG. 1. Time series (a) and PDFs (b) of a high-pass filtered density time series for 3 different cut-off frequencies, $f=10,50$ and $100 \mathrm{kHz}$. The dotted line corresponds to a Gaussian function.

different cut-off frequencies. It can be observed from this example how the PDFs become more peaked with higher cut-off frequency, that is to say there is a larger number of highamplitude events relative to what would be expected in the Gaussian case, and therefore a higher kurtosis. This indicates the presence of intermittency in the time series.

The presence of self-similar motion in the inertial range is a central assumption in Kolmogorov's 3D turbulence theory of 1941 (K41) 24,25 and Kraichnan's Kr67 theory for 2D turbulence ${ }^{26,27}$. Self-similar flows have no preferential direction and Gaussian statistics are observed over all scales of motion. A deviation from self-similarity implies intermittency, manifested by a deviation from Gaussian fluctuation statistics. By examining the kurtosis of signals high-pass filtered with different cut-off frequencies, the various scales of the signal are analysed. Note that skewness may not be used in this definition as self-similar turbu- 
lence can have a finite skewness ${ }^{7}$. In order to compare between different data sets using this method, the rate of increase of $K_{f}$ as a function of $f$ can be thought of as an indicator of the intermittency level (see discussion below).

For this analysis a wavelet transform (see for example ${ }^{28,29}$ ) was used instead of a highpass frequency filter. In this way, a signal is deemed intermittent if its kurtosis at the characteristic time scale $\tau, K_{1 / \tau}$, increases with the characteristic frequency $f=1 / \tau$, where the kurtosis is calculated from the wavelet coefficients $x_{\tau}(t)^{30,31}$, which are obtained from the wavelet transform

$$
x_{\tau}(t)=\frac{1}{\sqrt{\tau}} \int_{-\infty}^{+\infty} \psi\left(\frac{t-t^{\prime}}{\tau}\right) x\left(t^{\prime}\right) d t^{\prime} .
$$

The wavelet function, $\psi(t)$, used in the present analyses is the Morlet wavelet

$$
\psi(t)=C\left(e^{i \omega_{\psi} t}-e^{\omega_{\psi}^{2} / 2}\right) e^{-t^{2} / 2}
$$

where $C$ is a real constant. In order to provide a good trade-off between frequency and time resolution, the parameter $\omega_{\psi}$ was set to 6 . Results obtained using a high-pass filter are similar and are, therefore, not presented.

Another method, commonly used in the field of neutral fluid turbulence to determine the intermittency level of a data set is the method of structure function analysis (see for example Refs. $\left.{ }^{24,32,33}\right)$. For a variable $g(\mathbf{r})$ defined in space, the structure function of order $q$ for points spaced $L$ apart in the field is calculated from

$$
S_{q}(L)=\left\langle|g(\mathbf{r}+L)-g(\mathbf{r})|^{q}\right\rangle
$$

where the absolute value is taken to improve convergence ${ }^{13,34}$, and angular brackets indicate a spatial average. The scaling of the structure function with the spacing $L$ can be analysed to determine if intermittency is present. In the absence of intermittency, structure functions in the inertial range are expected to scale as $S_{q}(L) \propto L^{\zeta_{q}}$, where the exponent of the structure function $\zeta_{q}$ scales linearly with the order $q$. The factor of proportionality in this scaling is related to the slope of the energy spectrum in the inertial range. A deviation from linear behavior between $\zeta_{q}$ and $q$ is an indicator of intermittency. The accuracy of measuring $\zeta_{q}$ is greatly increased by the presence of extended self-similarity (ESS), resulting in an extended region of linear scaling when $S_{q}$ is plotted against $S_{3}$ instead of $r$ on a log-log scale ${ }^{15,35}$. The 
method assumes that any "undulations" 24 in structure functions, stemming for example from possible measurement issues, impact all structure functions in a similar way, and can therefore be overcome by comparing the structure functions against $S_{3}$. This observation allows a more accurate estimate of $\zeta_{q}$ to be made, and therefore a more accurate estimate of the scaling of the power-law relation, and the intermittent properties of the data. The intermittency level may be estimated by calculating the deviation of the 6th order structure function scaling exponent $\zeta_{6}$ from the non-intermittent prediction of $\zeta_{6} / \zeta_{3}=2^{24,32}$. This is sometimes called the intermittency exponent ${ }^{24,32}$, and it appears in the log-normal model of intermittency. Using this as a measure for intermittency in our case makes additional sense since it makes use of the highest available experimental structure function order, which deviates the most from the non-intermittent prediction. In experiments, spatial information with sufficient resolution is often not available so time series are analysed instead. This requires that the Eulerian perspective of the flow field is studied, and structure functions are calculated from separations in time, $\tau$, instead of space, $L, S_{q}(\tau)=\left\langle|x(t+\tau)-x(t)|^{q}\right\rangle$, where $x(t)$ may be any experimentally accessible time series, $n_{e}(t), \phi(t)$ etc. This is made

possible by invoking Taylor's hypothesis ${ }^{23}$, which states that the turbulence can be thought of as "frozen" as long as the mean flow is much greater than the turbulent eddy velocity, implying that the spatial properties of the turbulence may be deduced from measurements of time series at a fixed location. By measuring the plasma potential in TJ-K, it can be shown that the fluctuating $E \times B$ velocity is around 3-4 times smaller than the mean flow at the inner edge of the separatrix.

\section{THE TORSATRON TJ-K AND DIAGNOSTICS}

Experiments were conducted at the torsatron TJ-K ${ }^{17}$, a small-sized $l=1$ stellarator with a major radius of $0.6 \mathrm{~m}$ and a minor radius of $0.1 \mathrm{~m}$. The magnetic flux surfaces have 6 fold symmetry, and are generated by a single helical winding and two vertical field coils, as depicted in Fig. 2. For the experiments discussed here, plasma ionisation and heating were performed using a $2.45 \mathrm{GHz}$ magnetron ( $\mathrm{see}^{36}$ for a discussion of plasma heating in TJ-K). Poloidal limiters were used at two toroidal locations (see Fig. 2) to create a region of uniform connection length close to the natural last closed flux surface (LCFS), resulting in a more homogeneous edge region. Typical electron densities are $n_{\mathrm{e}}=5 \times 10^{17} \mathrm{~m}^{-3}$ with 


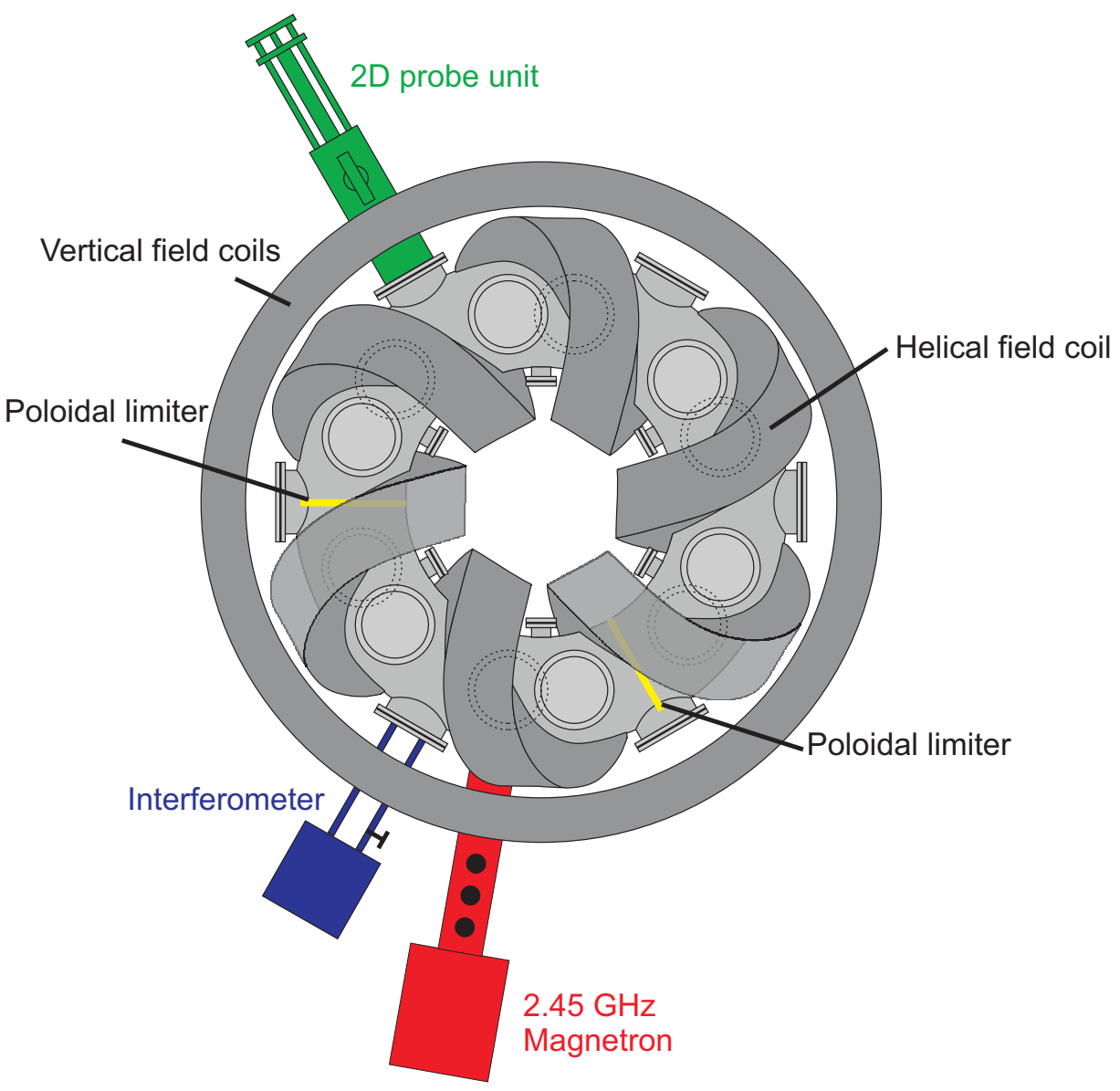

FIG. 2. A top-view schematic diagram of the torsatron TJ-K showing the coil arrangement and key diagnostics.

typical electron temperatures of $T_{\mathrm{e}}=10 \mathrm{eV}$ and cold ions, $T_{\mathrm{i}}<1 \mathrm{eV}{ }^{18}$. Turbulence in TJ-K is drift-wave dominated ${ }^{20-22}$, and dimensionless parameters governing edge turbulence are similar in TJ-K plasmas to those found in the edge region of fusion devices ${ }^{17,20}$, making results obtained in TJ-K of interest to the wider fusion community.

Due to the low temperatures and densities of TJ-K plasmas, Langmuir probes may be used throughout the entire plasma volume allowing relatively long time series of electron density, $n_{\mathrm{e}}$, and plasma potential, $\phi_{\mathrm{pl}}$, fluctuations to be recorded with high temporal resolution and good spatial resolution. Fluctuations in electron density are proportional to fluctuations in the ion saturation current drawn by a biased probe due to the lack of coherent temperature fluctuations in $\mathrm{TJ}-\mathrm{K}^{37}$. For the same reason, fluctuations in the floating potential are proportional to the plasma potential fluctuations. The data acquisition rate for all time series measurements presented here was $1 \mathrm{MHz}$, and time series were typically 


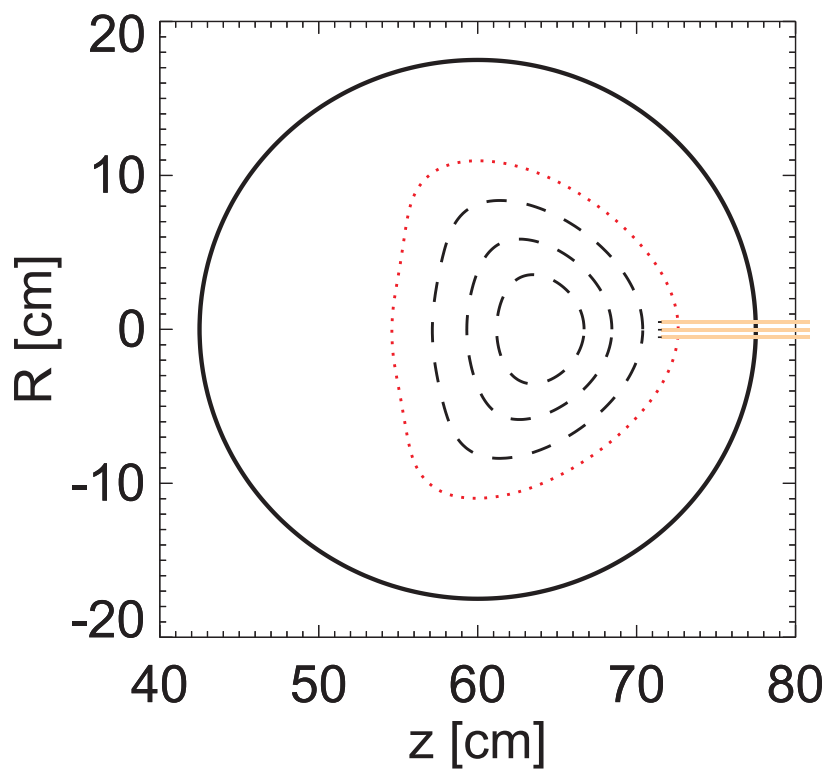

FIG. 3. A schematic diagram of the positioning of the Langmuir probes in the measuring poloidal cross-section. Flux surfaces are given by dashed lines and the last closed flux surface is given by the red dotted line.

$\approx 0.5 \mathrm{~s}$ in duration, providing a large enough sample to study higher order structure functions. Typical auto-correlation times are on the order of $10 \mu \mathrm{s}^{21}$. The probe measurements were performed with a $2 \mathrm{D}$ probe unit (see Fig. 2 for the location of the probe system), capable of measurement in a poloidal cross-section of the plasma, depicted in figure 3. For the measurements presented here 3 probes were necessary; to measure $n_{\mathrm{e}}, \phi_{\mathrm{pl}}$, as well as the electron temperature, $T_{\mathrm{e}}$ obtained from the IV-characteristic of a swept probe. For this study, half radial profiles were obtained in order to correctly determine density profiles, and therefore the collisionality, however the fluctuation data presented was obtained exclusively at a radial location at the mid-plane, approximately $1 \mathrm{~cm}$ inside the LCFS. Figure 3 shows a diagram with the position of the 3 Langmuir probes relative to the flux surface configuration in the poloidal cross-section. Due to the relatively steep density gradient, the fluctuation amplitude of drift-wave turbulence is expected to be highest in this region, making it the obvious choice for a systematic study of the intermittent properties of drift-wave turbulence.

In the case where electron-electron collisions dominate electron parallel mobility, the collisionality, $C$, may be calculated from the relation 


$$
\begin{aligned}
C & =\frac{\nu_{\mathrm{ee}} / \omega_{\mathrm{ce}}}{\left(k_{\|} \rho_{\mathrm{s}}\right)^{2}} \\
& =\frac{n_{\mathrm{e}} m_{\mathrm{e}} B e}{1.4 \times 10^{10} m_{\mathrm{i}} k_{\|}^{2} T_{\mathrm{e}}^{5 / 2}},
\end{aligned}
$$

where $m_{\mathrm{e}}$ and $m_{\mathrm{i}}$ are the electron and ion masses, $B$ is the magnetic field strength, and $e$ the electron charge. The electron-electron collision frequency is given by $\nu_{\mathrm{ee}}=n_{\mathrm{e}} /(1.4 \times$ $10^{10} T_{\mathrm{e}}^{3 / 2}$ ), the electron cyclotron frequency by $\omega_{\mathrm{ce}}=e B / m_{\mathrm{e}}$, and the drift scale is given by $\rho_{\mathrm{s}}=\sqrt{m_{\mathrm{i}} T_{\mathrm{e}}} /(e B)$. The electron-electron collision frequency is the relevant quantity in TJ-K plasmas since it dominates the electron-neutral and electron-ion collisionalities. In order to determine the local collisionality from Eq. 5, it was necessary to measure the electron temperature as well as the local density. The electron temperature was deduced from the characteristics of swept probes. Density profiles were obtained from radial profile probe measurements calibrated using the line averaged density, obtained using a $64 \mathrm{GHz}$

microwave interferometer. The parallel wave numbers, $\hat{k}_{\|}$, for $\mathrm{H}$, He, and Ar were previously calculated in Ref. 38 , and the values for $\mathrm{D}$ and Ne were determined from linear interpolation with existing data. In order to vary the collisionality, different ion species were used, and scans of the neutral gas pressure and heating power were made. Lighter ions are associated with higher collisionalities, which is clear when considering the ion mass in the denominator of Eq. 5. Higher neutral gas pressures also tend to result in higher collisionalities due to an increased electron density, whereas no clear trend is observed between collisionality and microwave heating power. The collisionalities obtained from these experiments span approximately the range $C=0.1$ to 12 , allowing for detailed study of scaling of intermittency level with collisionality.

\section{HASEGAWA-WAKATANI SIMULATIONS}

Fluctuations in the edge region of magnetically confined fusion plasmas are commonly dominated by drift-wave turbulence ${ }^{39}$, which occurs as a result of the combined destabilising effect of magnetic field curvature and resistance to parallel electron motion in a pressure gradient for a plasma immersed in a strong magnetic field. The Hasegawa-Wakatani (HW) model $^{8,9}$ constitutes a relatively simple two-field model able to capture the essence of collisional drift-wave turbulence. A large amount of research exists employing the HW model 
to study the statistical properties of drift wave turbulence (see for example ${ }^{10,12,14,16}$ ). The equations for the two-dimensional slab version of the model with isothermal electrons and cold ions can be written in dimensionless form as

$$
\begin{aligned}
\partial_{t} n-\{\phi, n\}+\partial_{y} \phi \frac{1}{L_{n}} & =\frac{1}{C}(\phi-n) \\
\partial_{t} \Omega-\{\phi, \Omega\} & =\frac{1}{C}(\phi-n),
\end{aligned}
$$

where $n$ is the density, $\phi$ the potential, $\Omega=\nabla^{2} \phi$ the vorticity and $L_{n}$ the density gradient. The Poisson bracket is defined as $\{A, B\}=\partial_{x} A \partial_{y} B-\partial_{x} B \partial_{y} A$. Artificial damping terms of the form $\nu \nabla^{2} n$ and $\mu \nabla^{2} \Omega$ (Newton viscosity) are added to the right hand side of Eqs. 6, effectively damping high wavenumbers and providing an energy and enstrophy sink. The parameter $C$ is the collisionality, governing the parallel motion of the electrons, and is given by Eq. 5 in the case of TJ-K. In the HW model, damping of the flow occurs due to the parallel resistivity, described by the dimensionless $C$ parameter. The flow is determined from the plasma potential, and is equal to the $E \times B$ velocity, where $E$ is the electric field given by $\mathbf{E}=-\nabla \phi$, and $\mathbf{B}$ is the magnetic field. The $\phi$ parameter acts, therefore, as the stream function.

For the purpose of this work simulations were carried out with varied collisionality, in order to test for trends observable in the intermittency of the density and potential fluctuations, which are relatively easily accessed experimental quantities. The runs were performed on a $40 \times 40 \rho_{\mathrm{s}}$ grid with $512 \times 512$ nodes, and the time step was chosen to be $0.005 \rho_{\mathrm{s}} / c_{\mathrm{s}}$, where $c_{\mathrm{s}}=\sqrt{T_{\mathrm{e}} / m_{\mathrm{i}}}$ is the ion speed of sound. Artificial damping was Newtonian, and set to $\nu=\mu=0.01$. After a period of linear growth the energy and enstrophy saturate (see figure 4), and non-linear terms come to dominate. Following this point data may be taken to analyse the intermittency level.

For the analysis of intermittency, snapshots of $n, \phi$ and $\Omega$ were taken over a period of 50000 time steps, with a snapshot output for analysis every 500 time steps. The eddy turnover time, $t_{e}$, is between 120 and 160 time steps, where $t_{e}$ is calculated from $t_{e}=1 / \sqrt{W}$, with $W$ being the rms vorticity ${ }^{12}$. Figure 5 shows snapshots of the three fields in the saturated regime for collisionalities $C=0.67,1.25$ and 5 . It can clearly be observed how the morphology of the density field changes going from low collisionality $(C=0.67)$, where the density strongly resembles the potential, to the higher collisionality case $(C=5)$, where 

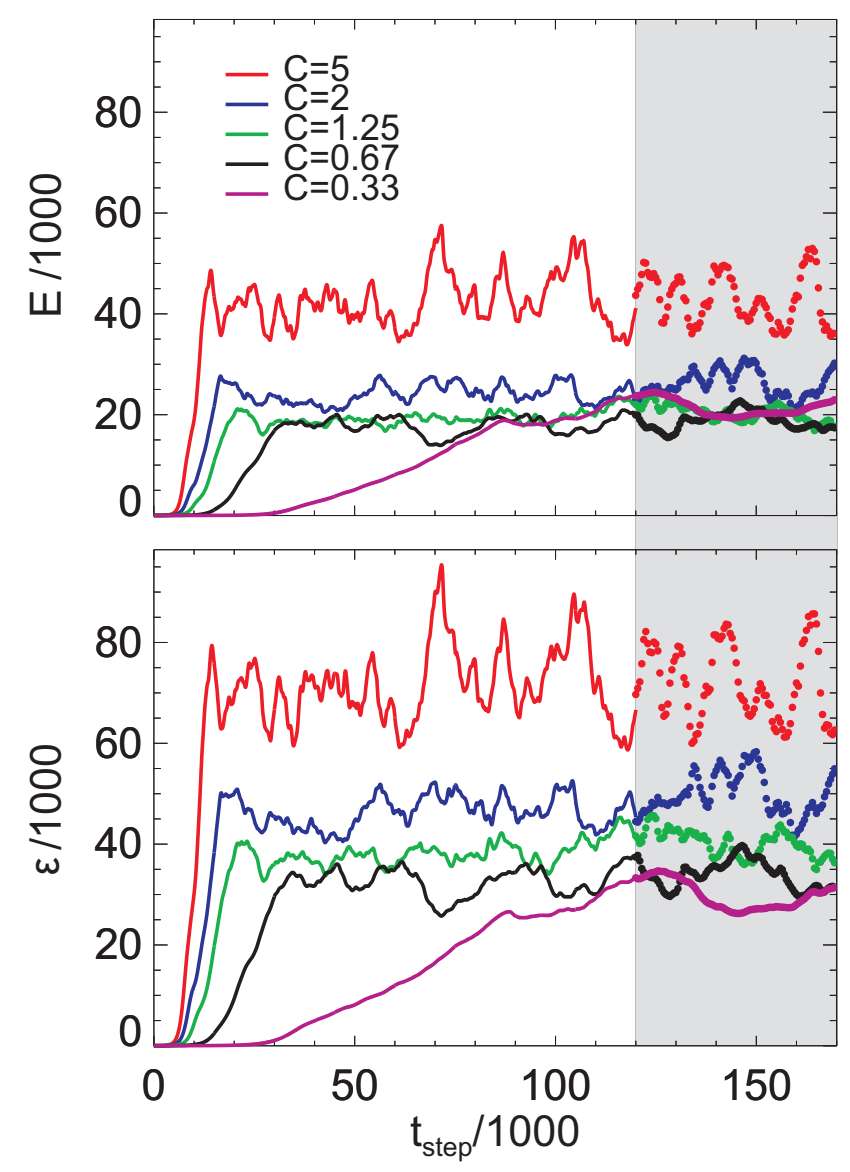

FIG. 4. The energy, $E$, and enstrophy, $\epsilon$, integrated over the simulation grid as a function of simulation time step, $t_{\text {step }}$ for the 5 collisionalities investigated, $C=0.33,0.67,1.25,2$ and 5 . The grey shaded area indicates the simulation times over which data was analysed.

the density field is more consistent with that of the vorticity. This can be understood by considering the HW equations given by Eqs. 6. When $C$ is small, the coupling between $n$ and $\phi$ is large since there is little resistance to the parallel electron motion and electrons can react quickly to perturbations in the density. When $C$ is large, the coupling parameter $(\phi-n) / C$ becomes small. In this case, the density perturbations do not resemble the potential, but instead evolve in the sense of a passive scalar, taking on the morphology of the vorticity. This behaviour can also be observed in the power spectra from the same simulation runs, displayed in Fig. 6.

The intermittency properties of $n, \phi$ and $\Omega$ can be compared for different collisionalities using the spatial structure function analysis described in section II. Here the statistical average was taken over the entire simulation grid and the 100 snapshots of the fields taken 

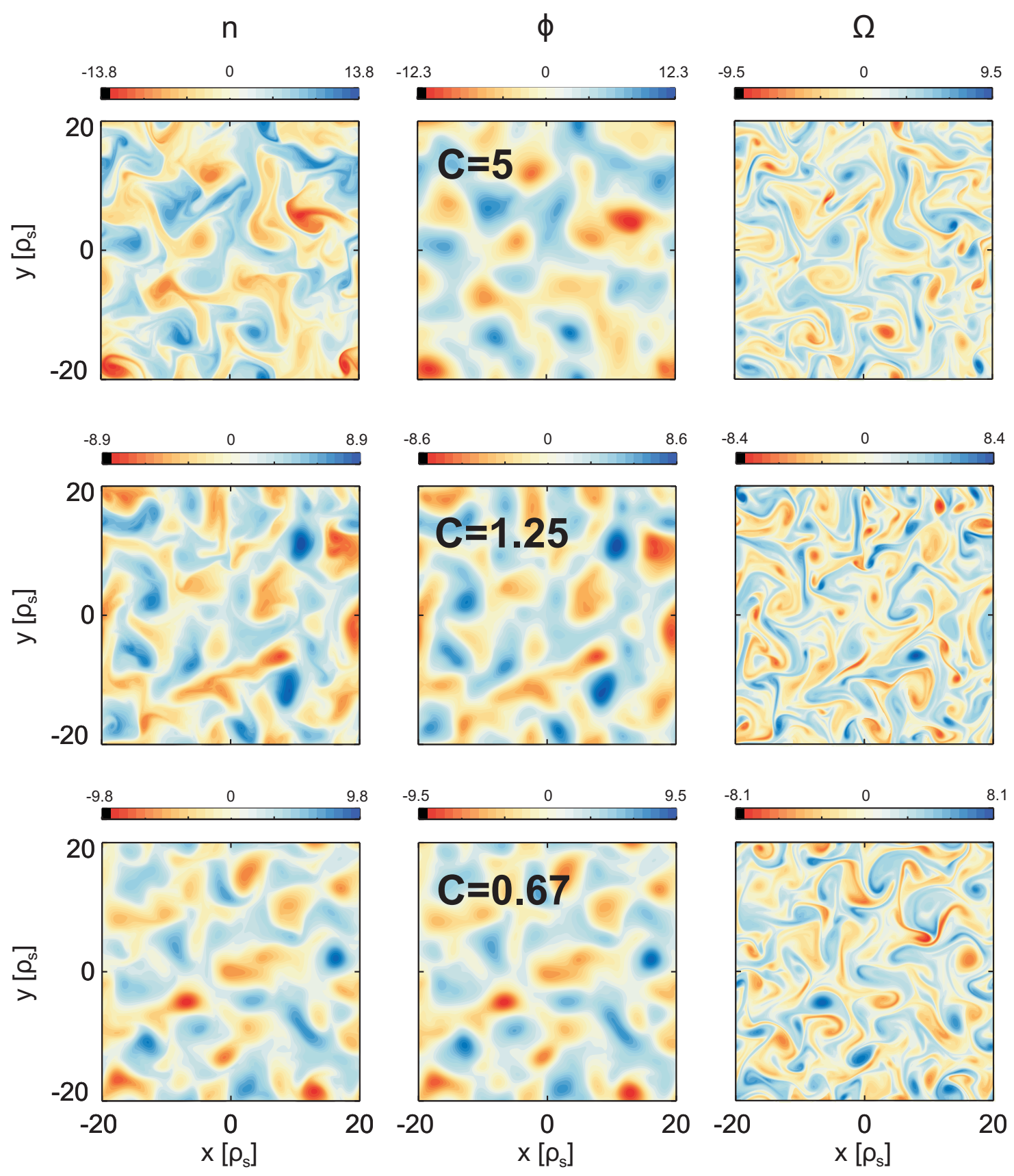

FIG. 5. Snapshots of the density, $n$, potential, $\phi$, and vorticity $\Omega$ fields at three different values of the collisionality, $C=0.67,1.25$ and 5 .

after energy and enstrophy have saturated. The quantity of data collected is sufficient to calculate up to the 6th order structure function, determined using the method provided in Ref. ${ }^{40}$. Figure 7a) shows the structure functions for the density field up to order $q=6$ as a function of the distance between points in the field, L. Using the ESS technique, plotting the structure functions against the 3rd order structure function, the range over which a 

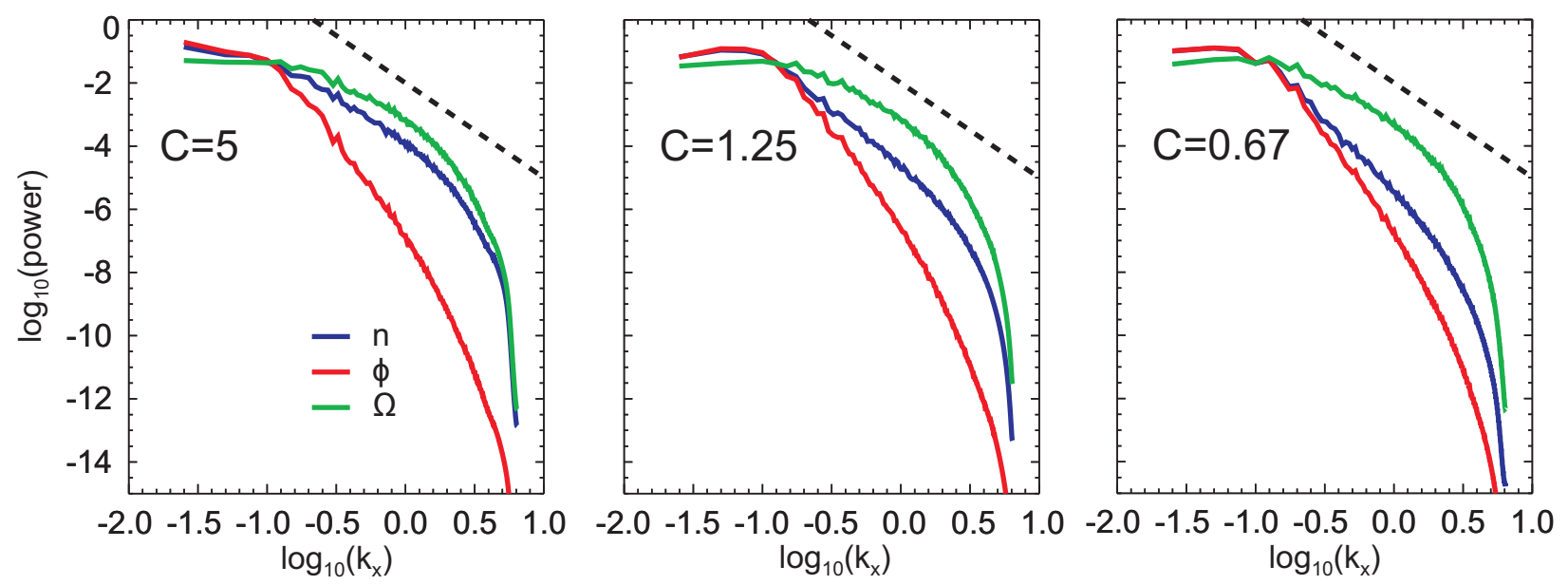

FIG. 6. Power spectra for the density, $n$, potential, $\phi$, and vorticity $\Omega$ fields at three different values of the collisionality, $C=0.67,1.25$ and 5 . The dashed line indicates the slope $k^{-3}$.

power-law relation is observed increases, as shown in Fig. 7b), where the black line is a linear fit to the data points. For the ESS analysis, data was taken from the smallest scales up to the beginning of the saturation region (at $L \approx 5$ in Fig. 7 ), however the fit in the ESS analysis is not strongly affected by the inclusion of larger spatial scales. Fit ranges which do not go up to the saturation region generally result in slightly steeper gradients in the ESS plots, meaning lower levels of intermittency. The resulting gradient does not, however, vary greatly, with a maximum increase in $\zeta_{6} / \zeta_{3}$ of approximately 0.02 for the vorticity, which exhibits the largest change. This allows the exponent $\zeta_{q}$ to be determined from the gradient of the linear fit on a $\log -\log$ plot, $\zeta_{q} / \zeta_{3}=\log _{10}\left(S_{q}\right) / \log _{10}\left(S_{3}\right)$. The normalised structure function exponents for $n, \phi$ and $\Omega$ are shown as a function of order $q$ for the same data set in Fig. 8, corresponding to a simulation run with $C=1.25$. When no intermittency is present, $\zeta_{q} / \zeta_{3}$ is expected to follow a linear relationship with $q$, as indicated by the dashed line, $\zeta_{q} / \zeta_{3}=q / 3$. In the case of $\phi$, the structure function exponents follow the dashed line closely up to $q=6$, with only a slight deviation at higher orders. For $n$ and $\Omega$, however, the curves deviate from a linear relationship at orders $q>4$, indicating the presence of intermittency.

As mentioned in section II, the level of the intermittency may be characterised by the deviation of 6th order structure function exponents from the non-intermittent prediction at $q=6$. This facilitates comparison of the intermittency level between simulation runs. Fig. 9 shows the intermittency level, $2-\zeta_{6} / \zeta_{3}$, for $n, \phi$ and $\Omega$ as a function of collisionality. All 

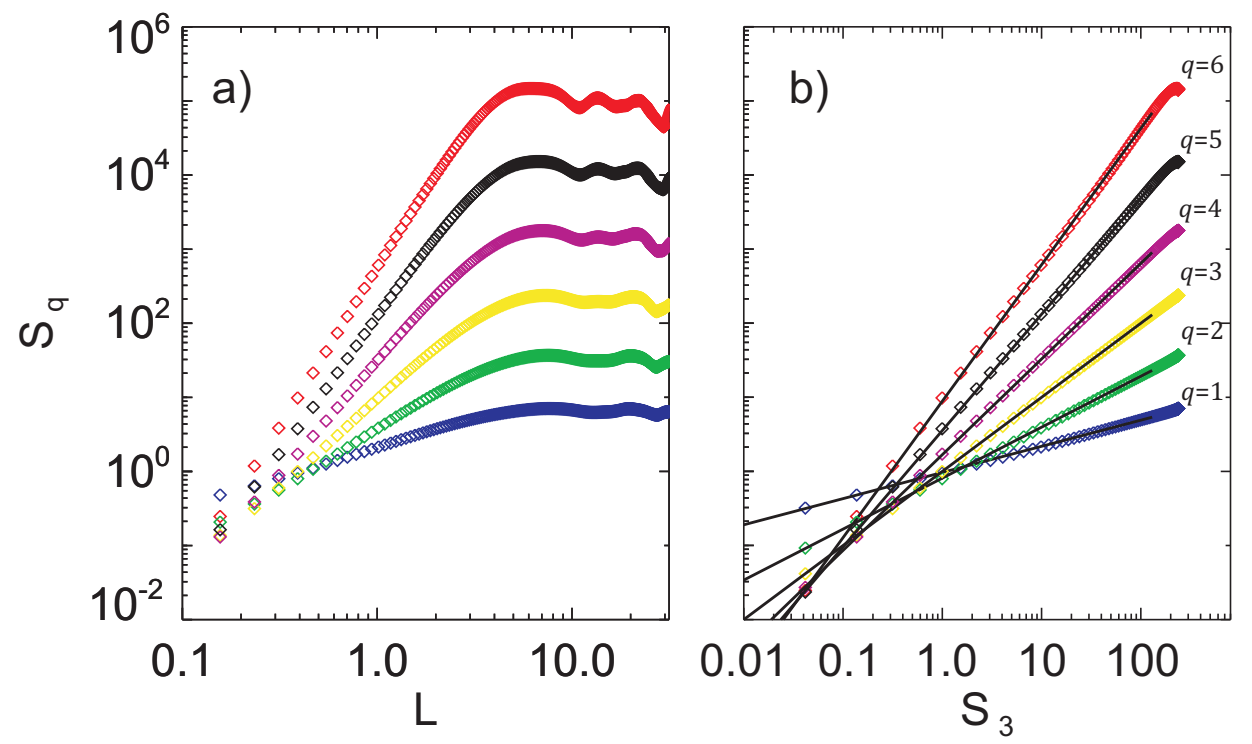

FIG. 7. a) Example density structure functions, $S_{q}$, up to $q=6$ as a function of the distance between points, $L$. Structure functions were obtained by averaging the moments over space and time. b) The ESS analysis for the same data, where the solid black line is a linear fit $(C=1.25)$.

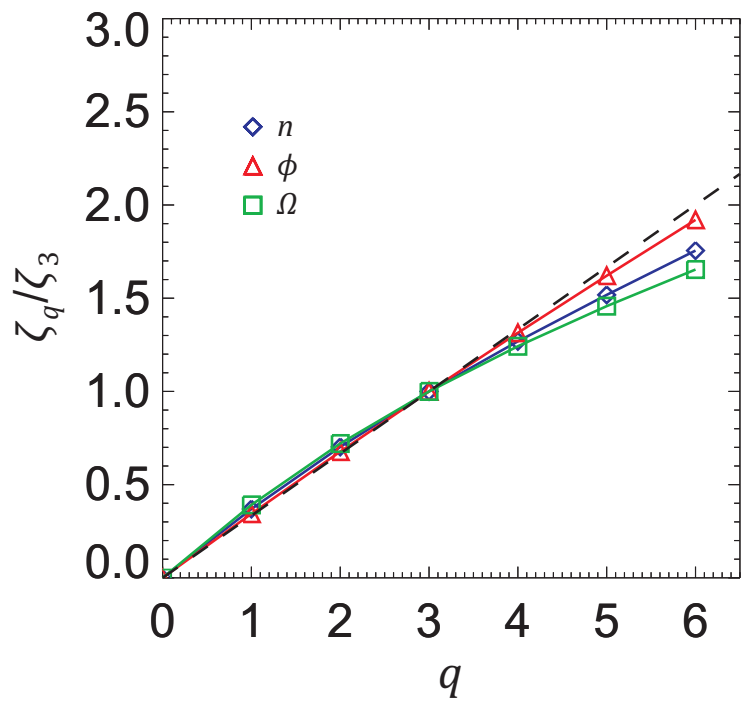

FIG. 8. The normalised structure function exponent $\zeta_{q} / \zeta_{3}$ as a function of the order $q$ for $n, \phi$ and $\Omega(C=1.25)$. The dashed line represents the non-intermittent case of $\zeta_{q} / \zeta_{3}=q / 3$.

parameters were kept constant in the simulations apart from the collisionality. It can be seen from the figure that the potential fluctuations remain self-similar over the range of $C$ considered. The lack of intermittency in $\phi$ is in line with other HW simulations ${ }^{13,14}$. The vorticity, on the other hand, exhibits intermittent fluctuations over the entire range of $C$ 


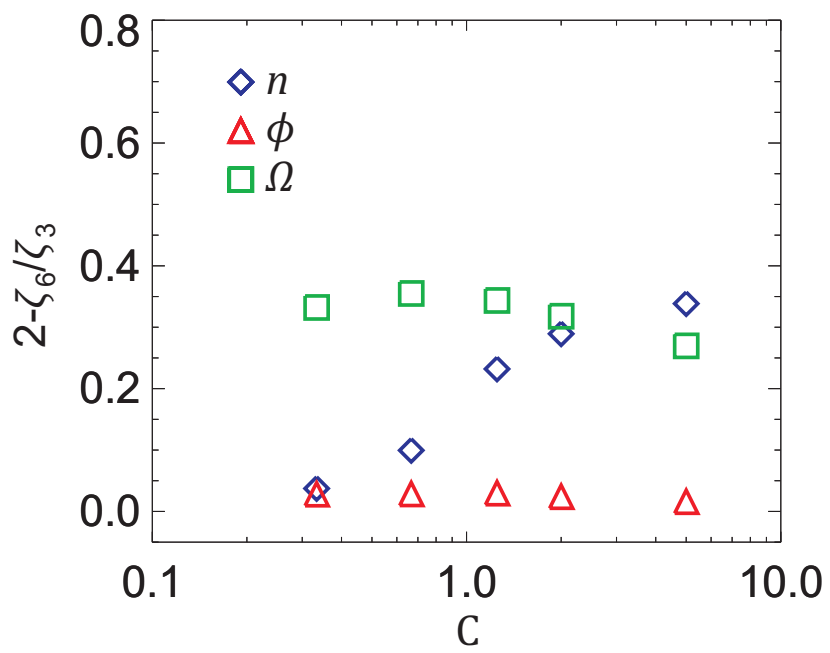

FIG. 9. The intermittency level, measured as $2-\zeta_{6} / \zeta_{3}$, as a function of collisionality for density, $n$, potential, $\phi$, and vorticity, $\Omega$ from Hasegawa-Wakatani simulations.

considered, with an approximately constant level. Intermittency of $\Omega$ has also been observed previously in HW simulations ${ }^{13,14}$. The density fluctuations, however, undergo a transition from being self-similar to more intermittent as the collisionality is increased. This can be understood in the framework of the HW model (equations 6) by considering how the densitypotential coupling changes as a function of $C$. At low $C$, going towards the adiabatic limit, the density-potential coupling is strong and the density fluctuations tend to closely follow the potential fluctuations (see the lower plots of Fig. 5), resulting in the statistics of the density fluctuations matching closely those of the potential. Going towards the hydrodynamic limit, at higher $C$, the coupling parameter becomes less important resulting in a density field which behaves more similarly to the vorticity (see the upper plots of Fig. 5), advected in a similar way to a passive scalar. Since the intermittency level of $\Omega$ does not change significantly as a function of $C$, the intermittency level of the density increases from the selfsimilar case of the potential, to the more intermittent case of the vorticity. This transition in the statistical properties of the density fluctuations as the collisionality in the plasma is increased is investigated experimentally, from two perspectives, in the next section. 


\begin{tabular}{|c|c|c|c|}
\hline working gas & $\mathrm{C}$ & $c_{s}\left[\mathrm{kms}^{-1}\right]$ & $\tau_{c}[\mu \mathrm{s}]$ \\
\hline \hline $\mathrm{H}$ & $5.3-24.8$ & $15.7-23.0$ & $2.4-3.6$ \\
\hline $\mathrm{D}$ & $2.2-13.4$ & $12.4-16.9$ & $3.3-4.5$ \\
\hline $\mathrm{He}$ & $0.5-2.4$ & $11.2-14.0$ & $4.0-5.0$ \\
\hline $\mathrm{Ne}$ & $0.1-0.6$ & $4.7-5.7$ & $9.9-12.0$ \\
\hline $\mathrm{Ar}$ & $0.1-0.4$ & $2.8-3.4$ & $16.4-19.7$ \\
\hline
\end{tabular}

TABLE I. The ranges of the parameters $C, c_{s}$ and $\tau_{c}$ covered by each working gas.

\section{EXPERIMENTAL RESULTS}

\section{A. Scale dependent kurtosis analysis}

An example plot of the kurtosis of density wavelet coefficients $n_{e, \tau}(t)$ versus the characteristic frequency $f=1 / \tau$, determined using the continuous wavelet transform, are presented in Fig. 10a). The data shown corresponds to analyses of five ion saturation current time series, $I_{\mathrm{i}, \mathrm{sat}}(t)$, obtained with the five working gases $\mathrm{H}, \mathrm{D}, \mathrm{He}$, Ne and $\operatorname{Ar}\left(\operatorname{in} \mathrm{TJ}-\mathrm{K} \tilde{I}_{\mathrm{i}, \text { sat }} \propto \tilde{n}_{e}\right.$ ). The parameter ranges corresponding to each working gas are summarised in Table I. For each working gas 20 separate discharges were carried out with varied neutral gas pressures and heating powers, enabling variation in $n_{\mathrm{e}}$ and $T_{\mathrm{e}}$, and therefore variation in $C$. The errors on the collisionality have been calculated from the estimated measurement errors on the density and temperature, resulting in maximum error bars of 0.7 for $\mathrm{H}, 0.4$ for $\mathrm{D}, 0.06$ for He, 0.005 for Ne and 0.01 for Ar. These errors are generally not visible in the figures, and so have been excluded from the plots. For H, D and He, the kurtosis can be seen to increase with cut-off frequency, $f$. Note that the highest kurtosis values for $\mathrm{H}, \mathrm{D}$, and $\mathrm{He}(K>50)$ are not displayed and that for $f \leq 10 \mathrm{kHz}$ the kurtosis is close to zero for all discharges. The graph indicates that $\tilde{n}_{e}(t)$ for $\mathrm{H}, \mathrm{D}$, and He is intermittent for frequencies above $50 \mathrm{kHz}$ since the kurtosis rises with characteristic frequency, while $\mathrm{Ne}$ and $\mathrm{Ar}$ are self-similar over all accessible frequencies.

Due to a mass dependence of the typical time scales involved in drift-wave turbulence, the cut-off frequencies corresponding to the analysis with each working gas are made dimensionless using the characteristic timescale, $\tau_{\mathrm{c}}=L_{\mathrm{n}} / c_{\mathrm{S}}{ }^{41}$, where $L_{\mathrm{n}}$ is the density gradient scale length and $c_{\mathrm{S}}=\sqrt{T_{\mathrm{e}} / m_{\mathrm{i}}}$ is the sound speed. In Ref. ${ }^{41}$, the typical drift-wave turbulence 
spatial scale $\rho_{\mathrm{s}}$ is combined with the typical drift-wave propagation speed $v_{\text {dia }}$ to obtain the associated characteristic time scale $v_{d i a} / \rho_{\mathrm{s}} \approx c_{\mathrm{s}} / L_{n}$. Since $L_{\mathrm{n}}$ and $T_{\mathrm{e}}$ vary minimally in TJ$\mathrm{K}$, the frequency is mainly shifted according to the ion mass, as can be seen by comparing the inset plot in Fig. 10a) to that in Fig. 10b). On the normalised frequency scale, intermittency is generally observed in $\mathrm{H}, \mathrm{D}$, and He above $\tau_{\mathrm{c}} / \tau=0.2$ for the data taken here. Considering the trend of increasing collisionality with decreasing ion mass, a link between increasing collisionality and a more rapid increase in kurtosis with characteristic frequency can be made. If the rate of increase in kurtosis is taken to be an indicator of the degree of intermittency, then Fig. 10 suggests that a higher collisionality results in a signal that is more intermittent. This is not an unreasonable assumption since the more intermittent a signal is, the greater the deviation from Gaussian statistics would be expected to be at larger time scales (lower frequencies) as compared to a signal which exhibits little intermittency. In other words, a break from self-similarity would occur over a larger range of scales for high levels of intermittency than for lower levels of intermittency, extending further into the inertial range.

In order to investigate the link between collisionality and intermittency in a more systematic way, the kurtosis of the wavelet filtered density and potential signals, $n_{\mathrm{e}, \tau}(t)$ and $\phi_{\tau}(t)$, at $\tau_{\mathrm{c}} / \tau=0.6$ is plotted for every set of experimental input parameters, shown in Fig. 11a) and Fig. 11b) respectively. An approximately logarithmic increase of kurtosis with collisionality is observed for density fluctuations. Specifically, the degree of intermittency of density fluctuations increases with increasing collisionality above a threshold between $C=0.3$ and $C=1$. Whilst this general trend exists, within the individual working gases $\mathrm{He}, \mathrm{Ne}$ and $\mathrm{Ar}$ there is no tendency towards an increased intermittency level with increasing collisionality. In the case of helium, a certain level of intermittency is present, but this remains constant as a function of collisionality with $K \approx 9$. For neon and argon, however, no intermittency seems to be present at all from this analysis, and the data points cluster around $K=0$.

The scale separated kurtosis analysis of potential fluctuations, displayed in Fig. 11b), shows no discernible correlation between collisionality and intermittency level. Some data points have relatively high kurtosis values of up to $K=41$, indicating a high level of intermittency, however the majority of data points lie between $K=0$ and $K=12$. The reason for the apparent intermittency present in the potential fluctuations observed through this analysis is unclear, however it will be seen in the next section that using a structure 

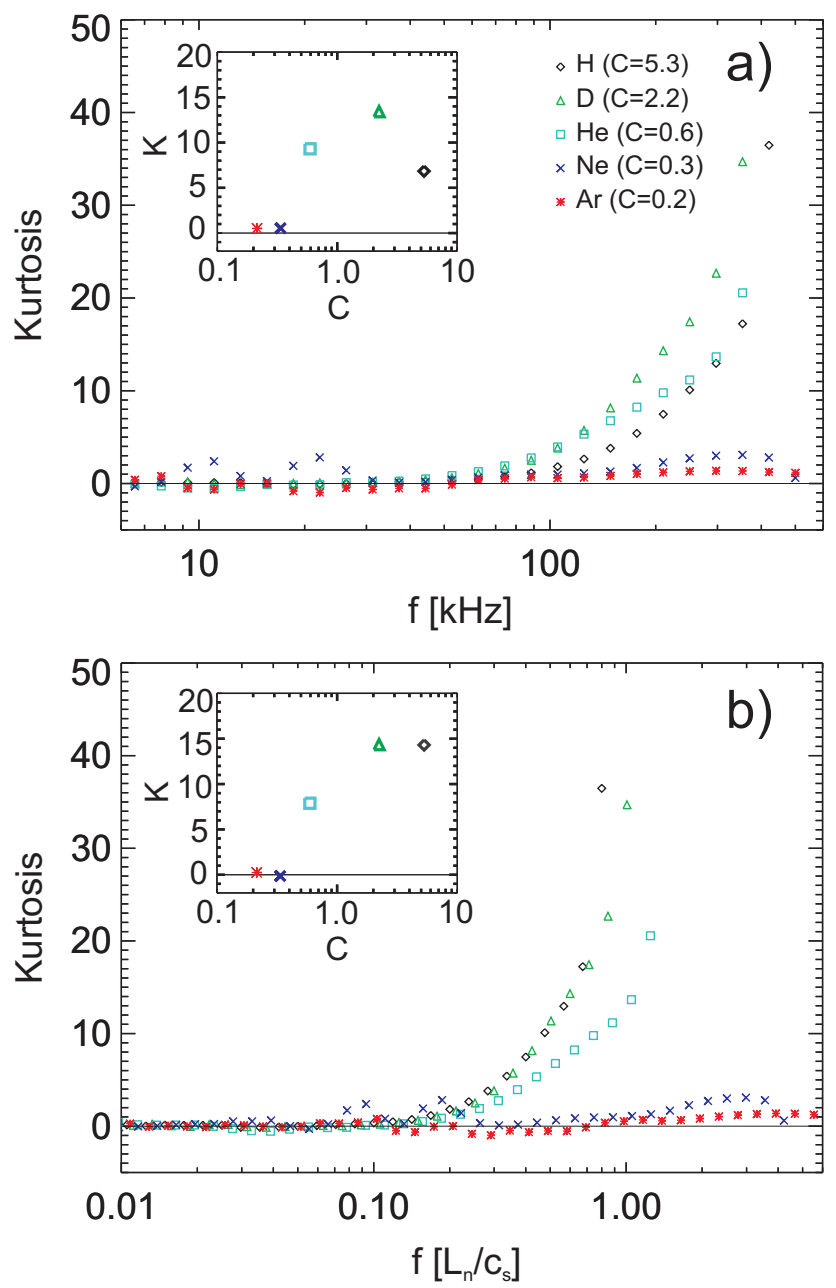

FIG. 10. a) The kurtosis of wavelet filtered density fluctuations as a function of characteristic frequency. The inset shows the kurtosis of each curve at $f=200 \mathrm{kHz}$ as a function of collisionality for the same data. b) The kurtosis of wavelet filtered density fluctuations as a function of the normalised characteristic frequency. The inset shows the kurtosis of each curve at $f L_{n} / c_{s}=0.6$ as a function of collisionality for the same data. Note that the highest frequency points for H, D, and He are not displayed.

function analysis, the intermittency does not appear to be present. In other experiments, potential fluctuations at the plasma edge were found to be self-similar (e.g. Ref. 42).

As mentioned in section IV the $n-\phi$ coupling becomes weaker as $C$ increases. It is therefore expected that below a certain threshold collisionality, the $n-\phi$ coupling is strong enough for the density field to be essentially self-similar. By decreasing the collisionality further still, no further decrease in $K$ can be expected for the density. This is likely the case 

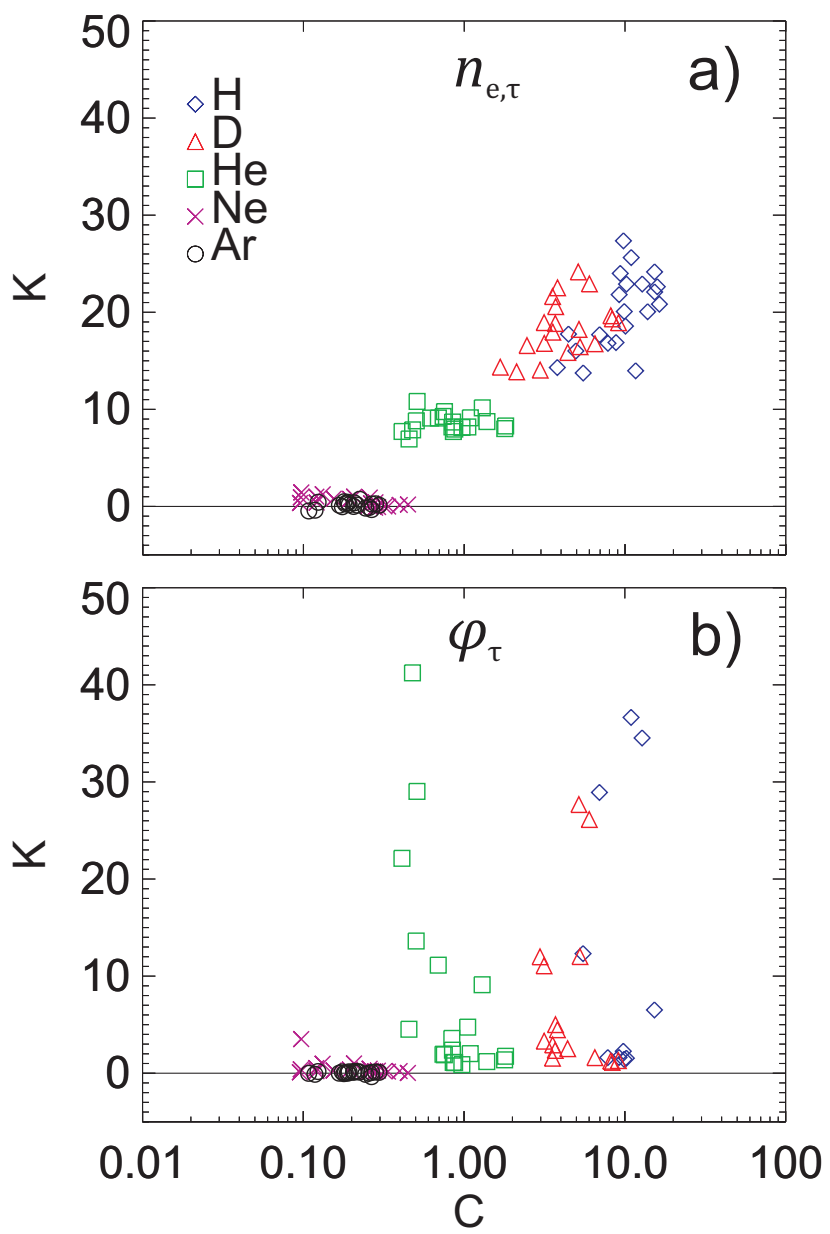

FIG. 11. The kurtosis of wavelet filtered density fluctuations, $n_{\mathrm{e}, \tau}(t)$, a) and potential fluctuations, $\left.\phi_{\tau}(t), \mathrm{b}\right)$ at a normalised frequency of $\tau_{\mathrm{c}} / \tau=0.6$, against the collisionality, $C$.

for Ne and Ar plasmas, due to their low collisionalities. The reason for no apparent decrease in $K$ with $C$ for the He plasmas is more puzzling, however there is reason to suspect that the effect of magnetic field curvature alters the $n-\phi$ coupling when $C \approx 1$, resulting in higher intermittency levels than those expected from the model used here. An extended HW model including a gradient in the magnetic field was used to show that in the region of bad curvature (corresponding to the measuring position for these experiments) the intermittency level of density fluctuations is higher compared to the case of a uniform magnetic field ${ }^{14}$. This effect should be most pronounced when the magnetic field inhomogeneity is of a similar order as the dimensionless collisionality.

The wavelet filtering method was implemented to give a more intuitive measure for the intermittency level, however the structure function analysis discussed in the next section 
provides a more robust method, and for this reason is the sole method used for the HW simulation data. The limitations of the wavelet method are two-fold. Firstly, the kurtosis determined from the plots in Fig. 10 is sensitive to the normalisation constant of the time scale due to the rapid increase of kurtosis with filter frequency for the hydrogen, deuterium and helium discharges at higher frequencies. A small uncertainty in this, due for example to uncertainty in the electron temperature measurement, can result in a relatively large uncertainty in the kurtosis value at a fixed $\tau_{\mathrm{c}} / \tau$. In addition, the method considers the turbulent statistics only at a fixed scale, whereas the structure function method uses a range of scales.

\section{B. Structure function analysis}

The calculation of high-order structure functions provides a more standard method for the analysis of intermittency in turbulent data series, and a useful comparison to the scale dependent kurtosis analysis given above. An example of structure functions calculated from $n_{e}(t)$ fluctuations in a helium discharge are shown in Fig. 12a). Saturation of the structure functions occurs at around $\tau>100 \mu s$. The $S_{q}(\tau)$ displayed in the figure are comparable to those observed in the plasma edge turbulence of other experiments ${ }^{43}$. As detailed in section II, the goal of the analysis is to determine the power law scaling of the structure function, $S_{q}(\tau) \propto \tau^{\zeta_{q}}$, if present, and find the exponent $\zeta_{q}$. A large power-law scaling region is not discernible in Fig. 12a). As with the HW simulation data in section IV, the method of extended self-similarity $(\mathrm{ESS})^{35,43}$, however provides an improved method for finding the region of power-law scaling. By plotting the structure functions against the 3rd order structure function, an extended region with a power-law type scaling is observed, as can be seen from Fig. 12b). Note that the saturation region at approximately $\tau>200 \mu \mathrm{s}$ is not displayed on the plot, and as with the simulated data, the fit was performed up to the saturation region. Using the ESS method, $\zeta_{q}$ normalised to the 3 rd order $\zeta_{3}$ can be easily calculated from the slope of the curve slope $=\log _{10}\left(S_{q}(\tau)\right) / \log _{10}\left(S_{3}(\tau)\right) \propto \zeta_{q} / \zeta_{3}$. In order to obtain convergent statistics for the structure function calculations ${ }^{40}$, the structure function order was limited to $q \leq 6$.

Example plots of $\zeta_{q} / \zeta_{3}$ against $q$ for $n_{e}$ and $\phi$ corresponding to shots with each of the working gases are shown in Fig. 13. The self-similar case is represented by the straight 

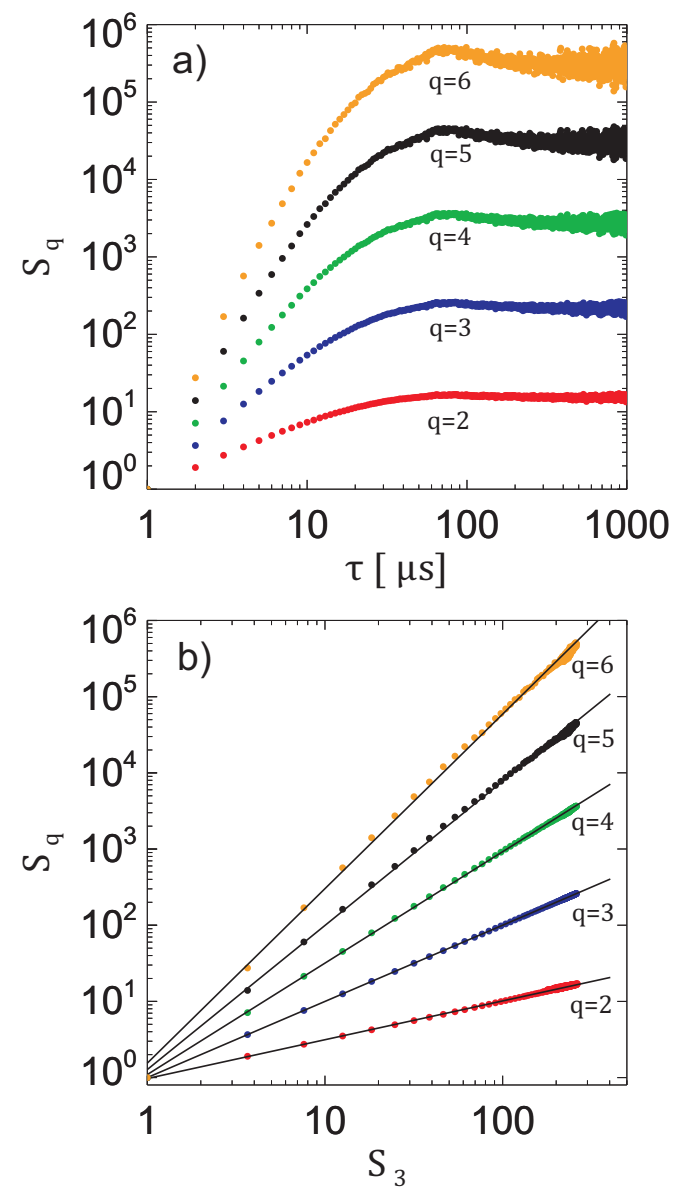

FIG. 12. Comparison of $S_{q}(\tau)$ scaling of $n_{e}$ fluctuations in a helium discharge with a) $\tau$ and b) $S_{3}(\tau)$. The $n_{e}$ fluctuations are normalised by the standard deviation $\sigma_{n}$, and $S_{q}(\tau)$ is normalised by its minimum value so that all curves are visible. The black lines in plot (b) are linear fits to the data.

dashed line and corresponds to the $\zeta_{q} / \zeta_{3}=q / 3$ prediction from Kolmogorov ${ }^{15,24}$. For the density fluctuations of the lighter gases taken for this example, the curve departs from a straight line for $q>4$, indicating intermittency. In addition, working gases with higher $C$ (lower ion masses) show a larger deviation from $q / 3$ than those with lower $C$, suggesting a similar trend with collisionality to that observed with the scale separated kurtosis analysis. For potential fluctuations, on the other hand, a linear scaling of the structure function exponent with order $q$ is observed up to the maximum order obtainable from the data, $q=6$, indicating that potential fluctuations are self-similar.

To quantify the level of intermittency, thereby allowing a comparison between discharges with different collisionalities, as with the HW data, the deviation of measured structure 

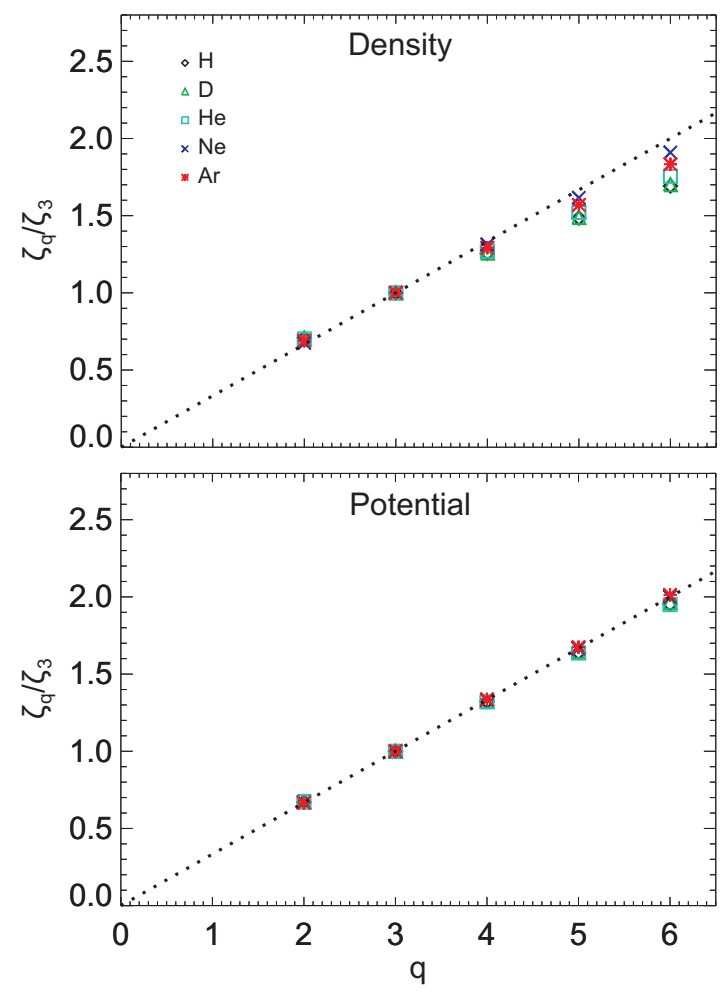

FIG. 13. Example plots of $\zeta_{q} / \zeta_{3}$ against $q$ for density $n_{\mathrm{e}}$ and $\phi$ calculated for each of the 5 working gases. The dotted line represents the non-intermittent case of $\zeta_{q} / \zeta_{3}=q / 3$.

function exponents of order 6 from the self-similar prediction of 2 may be used. The result of this analysis is shown in Fig. 14 for density and potential for the same data set as used in the scale separated kurtosis analysis, along with the results of HW simulations from section IV. The analysis shows a roughly similar trend to that observed from the scale separated kurtosis analysis, in that the intermittency level of density fluctuations, here represented by $2-\zeta_{6} / \zeta_{3}$, exhibits a general trend towards increasing intermittency with increasing collisionality. Interestingly, the trend shown by the HW simulations is remarkably similar, albeit offset from the experimental data at higher collisionality. This result suggests that the HW equations are capable of reasonably modelling the statistical properties of the TJ-K plasma, without accounting for phenomena which are excluded from the 2D slab HW model, such as zonal flows and magnetic field shear and curvature. In fact, it might be expected that the absolute values of the intermittency level would be closer to those experimentally observed if curvature were taken into account, since it has been demonstrated that an extended HW model, including an inhomogeneous magnetic field, results in higher intermittency level in the region of bad curvature ${ }^{14}$, corresponding to the 

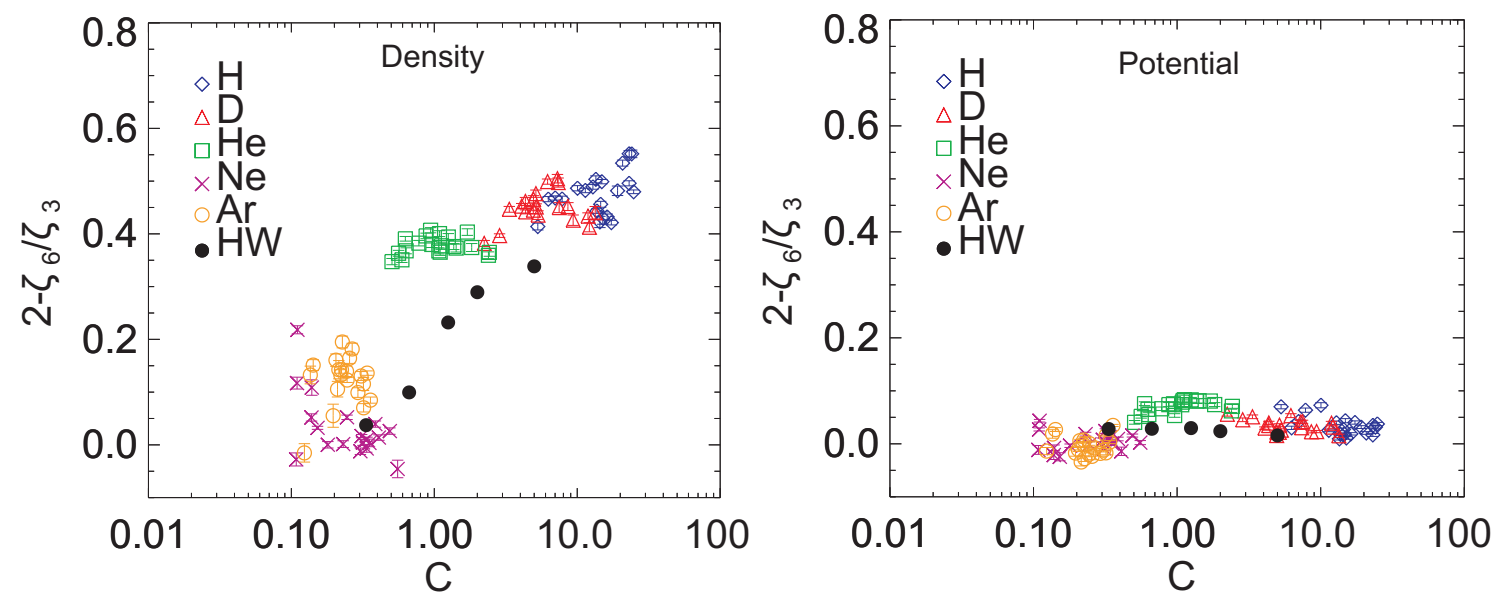

FIG. 14. The intermittency level $2-\zeta_{6} / \zeta_{3}$ against $C$ for $n_{\mathrm{e}}$ and $\phi$ from experimental time series data for $\mathrm{Ar}, \mathrm{Ne}, \mathrm{He}, \mathrm{D}$ and $\mathrm{H}$ as well as the intermittency level resulting from spatial structure functions calculated from Hasegawa-Wakatani simulations.

measurement region in these experiments. To more accurately simulate the TJ-K plasma, a 3D turbulence model would have to be implemented, taking into account full density and temperature profiles as well as the relatively complex stellarator magnetic field geometry, including the scrape-off layer. Such a model would, however, complicate the interpretation of the results, an issue which is simplified by using the $2 \mathrm{D}$ HW model where the $n-\phi$ coupling occurs only via the $C$ parameter.

Unlike the density fluctuations, the structure function analysis of potential fluctuations shows no increase in the level of intermittency with increasing collisionality. The results for both experimental and simulated data, displayed in the lower plot of Fig. 14, cluster around $2-\zeta_{6} / \zeta_{3}=0$. This result is similar to that found with the scale dependent kurtosis analysis and reinforces the presupposition that the points with higher kurtosis in Fig. 11b) are outliers due to limitations in the analysis method.

The increasing level of intermittency with collisionality for density fluctuations, and the absence of intermittency in potential fluctuations fits into the picture obtained from the Hasegawa-Wakatani model. By inspecting the snapshots shown in Fig. 5, it can be seen how the morphology of the density field changes as a function of $C$, from being more "smooth", and similar to the potential field, at low $C$, to having more fine scale structure at higher $C$, similar to the vorticity field. This can be understood by considering the HW model, Eqs. 6 , as described in section IV. 


\section{SUMMARY AND CONCLUSIONS}

The relationship between plasma collisionality and intermittency has been investigated for density and potential fluctuations in the stellarator TJ-K, as well as with HasegawaWakatani simulations. The intermittency level was determined in experiments using two methods. Firstly, the scale separated kurtosis analysis consisted of applying a wavelet transform to density and potential time series, and calculating the kurtosis of the wavelet coefficients at a chosen frequency scale ${ }^{30}$, similar to the method of calculating the kurtosis of a high-pass filtered signal ${ }^{24}$. Intermittency was characterised by the kurtosis at a fixed value of the characteristic frequency, normalised by a factor involving the density gradient scale length and the sound speed, of $f L_{\mathrm{n}} / c_{\mathrm{s}}=0.6$. A general trend of increasing intermittency level with increasing collisionality was observed for the density fluctuations, although kurtosis values for helium were approximately constant and no intermittency was observed for argon or neon. Potential fluctuations, on the other hand, were observed to be self similar with this analysis. Although some data points did exhibit higher levels of kurtosis, the general trend was towards low kurtosis values across the range of considered collisionalities.

The scaling of high-order structure functions was examined for the same experimental data set, along with the structure functions calculated from Hasegawa-Wakatani simulations. For density fluctuations, a general trend of increasing intermittency with increasing collisionality is observed, for both experimental data and simulated data. This trend agrees with the HW simulation result for the Lagrangian intermittency at high collisionality ${ }^{12}$. For potential fluctuations, on the other hand, no trend in the intermittency level and collisionality is observed, and the intermittency level remains low, indicating self-similar fluctuation statistics, in accordance with Refs. 13, 14, 44, and 45. This result suggests that potential fluctuations can be modelled using Gaussian statistics over the parameter range investigated.

The observed trend in intermittency level of density fluctuations with collisionality, and the self-similarity of potential fluctuations can be explained in the framework of the HW model. The model describes the spatio-temporal evolution of the density, potential and vorticity, with a parallel coupling term influenced by the collisionality. In the HW simulations, vorticity fluctuations have been found to be intermittent across the range of collisionalities used here. The result of intermittent vorticity is in agreement with other HW

simulations $^{13,14}$, and may be a general result for any passive scalar field ${ }^{46}$, which is the case 
for the vorticity equation in the high collisionality limit. At low collisionalities in the driftwave model, the resistance to parallel electron motion is low, allowing potential and density perturbations to closely follow each other. This is reflected in the HW model by a strong coupling between $n$ and $\phi$, and a strong similarity between the morphology of the two fields. Since the potential is self-similar regardless of the collisionality, in the low collisionality limit it is expected that the density field also exhibits low levels of intermittency. In the higher collisionality case, the coupling term becomes small and the density field acts more like a passive scalar in the flow field, similarly to the vorticity. The vorticity fluctuations from the Hasegawa-Wakatani simulations exhibit intermittency at all tested collisionalities. Since the density field comes to behave more like the vorticity field at high collisionality ${ }^{47}$, the density must also become more intermittent in this limit.

\section{REFERENCES}

${ }^{1}$ G. Birkenmeier, P. Manz, D. Carralero, F. M. Laggner, G. Fuchert, K. Krieger, H. Maier, F. Reimold, K. Schmid, R. Dux, T. Pütterich, M. Willensdorfer, E. Wolfrum, and The ASDEX Upgrade Team, Nuclear Fusion 55, 033018 (2015).

${ }^{2}$ D. A. D'Ippolito, J. R. Myra, and S. J. Zweben, Physics of Plasmas 18, 060501 (2011).

${ }^{3}$ D. Carralero, G. Birkenmeier, H. Müller, P. Manz, P. deMarne, S. Müller, F. Reimold, U. Stroth, M. Wischmeier, E. Wolfrum, and The ASDEX Upgrade Team, Nuclear Fusion 54, 123005 (2014).

${ }^{4}$ D. Carralero, P. Manz, L. Aho-Mantila, G. Birkenmeier, M. Brix, M. Groth, H. Müller, U. Stroth, N. Vianello, E. Wolfrum, ASDEX Upgrade team, JET Contributors, and EUROfusion MST1 Team, Physical Review Letters 115, (2015).

${ }^{5}$ T. Happel, F. Greiner, N. Mahdizadeh, B. Nold, M. Ramisch, and U. Stroth, Physical Review Letters 102, 255001 (2009).

${ }^{6}$ W. Horton, Physics Reports 192, 1 (1990).

${ }^{7}$ G. Boffetta and R. E. Ecke, Annual Review of Fluid Mechanics 44, 427 (2012).

${ }^{8}$ A. Hasegawa and M. Wakatani, Physical Review Letters 50, 682 (1983).

${ }^{9}$ M. Wakatani and A. Hasegawa, Physics of Fluids (1958-1988) 27, 611 (1984).

${ }^{10}$ S. J. Camargo, D. Biskamp, and B. D. Scott, Physics of Plasmas 2, 48 (1995).

${ }^{11}$ V. Naulin, New Journal of Physics 4, 28 (2002). 
${ }^{12}$ B. Kadoch, W. J. T. Bos, and K. Schneider, Physical Review Letters 105, 145001 (2010).

${ }^{13}$ S. Futatani, S. Benkadda, Y. Nakamura, and K. Kondo, Physics of Plasmas 15, 072506 (2008).

${ }^{14}$ P. D. Dura, B. Hnat, J. Robinson, and R. O. Dendy, Physics of Plasmas 19, 092301 (2012).

${ }^{15}$ V. P. Budaev, S. Savin, L. Zelenyi, N. Ohno, S. Takamura, and E. Amata, Plasma Physics and Controlled Fusion 50, 074014 (2008).

${ }^{16}$ W. J. T. Bos, B. Kadoch, S. Neffaa, and K. Schneider, Physica D: Nonlinear Phenomena 239, 1269 (2010).

${ }^{17}$ N. Krause, C. Lechte, J. Stöber, U. Stroth, E. Ascasibar, J. Alonso, and S. Niedner, Review of Scientific Instruments 73, 3474 (2002).

${ }^{18}$ S. Enge, G. Birkenmeier, P. Manz, M. Ramisch, and U. Stroth, Physical Review Letters 105, (2010).

${ }^{19}$ B. Schmid, P. Manz, M. Ramisch, and U. Stroth, Physical Review Letters 118 (2017), 10.1103/PhysRevLett.118.055001.

${ }^{20}$ U. Stroth, F. Greiner, C. Lechte, N. Mahdizadeh, K. Rahbarnia, and M. Ramisch, Physics of Plasmas 11, 2558 (2004).

${ }^{21}$ M. Ramisch, N. Mahdizadeh, U. Stroth, F. Greiner, C. Lechte, and K. Rahbarnia, Physics of Plasmas 12, 032504 (2005).

${ }^{22}$ N. Mahdizadeh, F. Greiner, T. Happel, A. Kendl, M. Ramisch, B. D. Scott, and U. Stroth, Plasma Physics and Controlled Fusion 49, 1005 (2007).

${ }^{23}$ G. I. Taylor, Proc. R. Soc. London, Ser. A 164, 476 (1938).

${ }^{24}$ U. Frisch, Turbulence: The Legacy of A. N. Kolmogorov (Cambridge University Press, Cambridge, 1995).

${ }^{25}$ A. N. Kolmogorov, Proceedings: Mathematical and Physical Sciences 434, 9 (1991).

${ }^{26}$ R. H. Kraichnan, Physics of Fluids 10, 1417 (1967).

${ }^{27}$ R. H. Kraichnan and D. Montgomery, Reports on Progress in Physics 43, 547 (1980).

${ }^{28}$ C. Torrence and G. P. Compo, Bulletin of the American Meteorological society 79, 61 (1998).

${ }^{29} \mathrm{~S}$. Mallat, A wavelet tour of signal processing: the sparse way (Academic press, Cambridge, 2008).

${ }^{30}$ N. Mahdizadeh, M. Ramisch, U. Stroth, C. Lechte, and B. D. Scott, Physics of Plasmas 
11, 3932 (2004).

${ }^{31}$ V. Carbone, G. Regnoli, E. Martines, and V. Antoni, Physics of Plasmas 7, 445 (2000).

${ }^{32}$ R. T. Cerbus and W. I. Goldburg, Physics of Fluids 25, 105111 (2013), 1306.0595.

${ }^{33}$ F. Anselmet, Y. Gagne, E. J. Hopfinger, and R. A. Antonia, Journal of Fluid Mechanics 140, 63 (1984).

${ }^{34}$ G. Y. Antar, P. Devynck, X. Garbet, and S. C. Luckhardt, Physics of Plasmas 8, 1612 (2001).

${ }^{35}$ R. Benzi, S. Ciliberto, R. Tripiccione, C. Baudet, F. Massaioli, and S. Succi, Physical Review E 48, R29 (1993).

${ }^{36}$ A. Köhn, G. Birkenmeier, A. Chusov, P. Diez, A. Feuer, U. Höfel, H. Höhnle, E. Holzhauer, W. Kasparek, S. Merli, M. Ramisch, J. Seifert, S. Wolf, and U. Stroth, Plasma Physics and Controlled Fusion 55, 014010 (2013).

${ }^{37}$ Z. Huang, Probe Measurement of Electron Temperature Dynamics in TJ-K and ASDEX Upgrade, Master's thesis, Erasmus Mundus Program on Nuclear Fusion Science and Engineering Physics (2011).

${ }^{38} \mathrm{~N}$. Mahdizadeh, Investigation of Three-Dimensional Turbulent Structures in the Torsatron TJ-K, Ph.D. thesis, Universität Stuttgart (2007).

${ }^{39}$ W. Horton, J. Kim, E. Asp, T. Hoang, T. Watanabe, and H. Sugama, AIP Conference Proceedings 1013, 1 (2008).

${ }^{40}$ T. Dudok de Wit, Physical Review E 70, (2004).

${ }^{41}$ P. Simon, M. Ramisch, A. A. Beletskii, A. Dinklage, M. Endler, S. Marsen, B. Nold, U. Stroth, P. Tamain, and R. Wilcox, Plasma Physics and Controlled Fusion 56, 095015 (2014).

${ }^{42}$ B. A. Carreras, B. P. Van Milligen, M. A. Pedrosa, R. Balbín, C. Hidalgo, D. E. Newman, E. Sánchez, M. Frances, I. García-Cortés, J. Bleuel, et al., Physics of Plasmas 5, 3632 (1998).

${ }^{43}$ V. P. Budaev, N. Ohno, S. Masuzaki, T. Morisaki, A. Komori, and S. Takamura, Nuclear Fusion 48, 024014 (2008).

${ }^{44}$ J. Paret and P. Tabeling, Physics of Fluids 10, 3126 (1998).

${ }^{45}$ G. Boffetta, A. Celani, and M. Vergassola, Physical Review E 61, R29 (2000).

${ }^{46}$ A. Celani, M. Cencini, A. Mazzino, and M. Vergassola, New Journal of Physics 6, 72 (2004). 
${ }^{47}$ P. Manz, M. Ramisch, and U. Stroth, Plasma Physics and Controlled Fusion 51, 035008 (2009). 